\title{
Lehrerkognitionen und die Gestaltung von Unterricht
}

\author{
Claudia Bergmüller · Ulrike Stadler-Altmann
}

\section{Sammelrezension zu}

1. Katharina Schwindt: Lehrpersonen betrachten ihren Unterricht. Kriterien für die kompetente Unterrichtswahrnehmung. Münster: Waxmann 2008. 197 S. Preis: 24,90€.

2. Ingelore Mammes: Denkmuster von Lehrkräften als Herausforderung für Unterrichtsentwicklung. Bad Heilbrunn: Klinkhardt 2008. 236 S. Preis: 29,90€.

3. Isabelle Hugener: Inszenierungsmuster im Unterricht und Lernqualität. Münster: Waxmann 2008. 262 S. Preis: 25,50€.

Unterrichtsentwicklung und die systematische Beschreibung von Unterricht sind zentrale Anliegen der pädagogischen Forschung. Wesentliches Ziel aktueller Forschungsansätze in diesem Kontext ist es, sowohl handlungsleitendes Wissen von Lehrkräften zu untersuchen und theorie- sowie empiriegeleitet zu rekonstruieren als auch Unterrichtsgestaltungen zu analysieren, die das Lernen der Schülerinnen und Schüler in besonderem Maße fördern.

In dieser Sammelrezension werden mit den Studien von Katharina Schwindt und Ingelore Mammes zwei Studien vorgestellt, die die Lehrperson und deren unterrichtsbezogene Kognitionen in den Blick nehmen. Die dritte Studie von Isabelle Hugener widmet sich dem konkreten Unterrichtsgeschehen. Alle drei Studien befinden sich im Zentrum der aktuellen Forschungsdiskussion zur Unterrichtsentwicklung und liefern Ergebnisse, die sowohl Hinweise für die Professionalisierung von Lehrkräften geben als auch Rückschlüsse auf Implementierungsmöglichkeiten von Entwicklungsprozessen in Schule und Unterricht ermöglichen.

\footnotetext{
Online publiziert: 16.01 .2010

Dr. C. Bergmüller $(\bowtie) \cdot$ PD Dr. U. Stadler-Altmann Lehrstuhl für Allgemeine Erziehungswissenschaft I Friedrich-Alexander-Universität Erlangen-Nürnberg Regensburger Straße 160, 90478 Nürnberg, Deutschland E-Mail: claudia.bergmueller@ewf.uni-erlangen.de

PD Dr. U. Stadler-Altmann

E-Mail: ulrike.stadler-altmann@ewf.uni-erlangen.de
}

(C) Die Autoren 2010. Dieser Artikel ist auf Springerlink.com mit Open Access verfügbar. 
Schwindt, Lehrpersonen betrachten ihren Unterricht. Katharina Schwindt untersucht in ihrer 2008 erschienenen Dissertation, über welche Kompetenzen Lehrpersonen in der Wahrnehmung von Unterrichtsaufzeichnungen verfügen und inwiefern sich Personen mit unterschiedlichem Erfahrungshintergrund in dieser Kompetenz unterscheiden (S. 149). Diese Kompetenzen sieht Schwindt als Indikator für das Potenzial von Lehrpersonen, im realen Unterrichtsgeschehen lehr-/lernrelevante Merkmale zu erkennen (S. 6). Es wurden Lehrpersonen mit unterschiedlichem Erfahrungshintergrund (Studierende, Lehrpersonen und Personen aus der Schulinspektion) gebeten, Unterrichtsaufzeichnungen unter strukturierten Aufgabenstellungen zu analysieren. Diese Bearbeitungen wurden inhaltsanalytisch ausgewertet und die Unterschiede zwischen den verschiedenen Gruppen von Lehrpersonen mit Hilfe univariater Varianzanalysen in den Blick genommen.

Schwindt knüpft in ihrer Untersuchung an wissensbezogene Konzepte der Professionalität von Lehrpersonen an, in denen der Wahrnehmung eine wichtige Vermittlungsfunktion zwischen Wissen und Handeln zugeschrieben wird. Dieser Zusammenhang wird in der Arbeit mithilfe des Konzepts der Schematheorie modelliert, nach der die Wahrnehmung als ein Interaktionsprozess zwischen bestehenden Wissensschemata und verfügbaren Informationen beschrieben wird. Die Bedeutung dieses Modells liegt für die Verfasserin in der Annahme, dass sowohl Wahrnehmungs- und Interpretationsprozesse von vorhandenen Wissensstrukturen beeinflusst werden als auch die Ergebnisse dieser Wahrnehmungsprozesse sich wiederum auf das Treffen von Handlungsentscheidungen auswirken (S. 38). Schwindt beschreibt Kompetenz in der Wahrnehmung als zentrale Vermittlungsgröße zwischen professionellem Wissen und Handeln. Die Kompetenz von Lehrpersonen in der Wahrnehmung von Unterricht wird damit in zweierlei Hinsicht relevant: (1) als „Indikator für verfügbares professionelles Wissen“ (S. 39) und (2) als „Grundlage für professionelles Handeln“ (ebd.).

Die Untersuchung von Katharina Schwindt ist eingebettet in die Studie „LUV - Lernen aus Unterrichtsvideos“, die von 2005 bis 2006 im Rahmen des DFG-geförderten Schwerpunktprogramms „BiQua - Bildungsqualität von Schule“, angesiedelt am Leibnitz-Institut für die Pädagogik der Naturwissenschaften (IPN) an der Universität Kiel, durchgeführt wurde. Die konkreten Fragestellungen des Projektes LUV sowie diejenigen der eigenen Untersuchung werden zu Beginn des theoretischen Teils der Arbeit (Kap. 2) umrissen. Im Anschluss daran begründet die Autorin vor dem Hintergrund verschiedener Traditionen zur Bestimmung von Lehrerprofessionalität die Bedeutung der angemessenen Wahrnehmung von Unterricht durch die Lehrpersonen (Kap.3). Im vierten Kapitel stehen Studien der Expertiseforschung im Fokus, die die Beschreibung von unterrichtsbezogenen Wahrnehmungskompetenzen von Lehrpersonen sowohl in ihrer jeweiligen Ausprägung als auch in ihrer Unterschiedlichkeit zum Gegenstand haben. Diese Studien werden von der Autorin im Hinblick auf ihre Aussagekraft kritisch diskutiert. Damit wird deutlich gemacht, was die eigene Arbeit in diesem Kontext leisten soll: „Es wird der Versuch unternommen, Kriterien für die kompetente Unterrichtsbeobachtung auf theoretischer Basis abzuleiten und Merkmale von Experten und Novizen in diese Systematik einzuordnen“ (S. 57). Anschließend werden Merkmale aus der Beobachtungsforschung auf die Wahrnehmung von Unterrichtsaufzeichnungen übertragen (Kap.5) und - angereichert durch empirische Ergebnisse aus den in Kap. 4 dargestellten Studien - Kriterien für die Kompetenzen von Lehrkräften im Hinblick auf diese Wahrnehmung abgeleitet. 
Hieraus entwickelt Katharina Schwindt ihr „Gesamtmodell der kompetenten Unterrichtswahrnehmung“ (Kap. 5.2.3). Vor diesem theoretischen Hintergrund werden im sechsten Kapitel die Fragestellungen der Untersuchung konkretisiert und das methodische Design der Studie (Kap. 7) beschrieben. In Kapitel acht widmet sich die Autorin den Ergebnissen ihrer Untersuchung. Dabei beginnt sie mit einer Darstellung der deskriptiven Befunde im Hinblick auf die Teilkompetenzen der Teilnehmerinnen und Teilnehmer ihrer Studie, ,die für eine vertiefende Analyse von Unterrichtsaufzeichnungen als zentral angesehen werden“ (S. 120) (Kap. 8.1.1). Der Fokus liegt auf folgenden fünf Teilkompetenzen: „Analyseprozess“, „Fokussiertheitsgrad der Analyse“, „Umfang und Art der Klassifikation“, „Qualität der schriftlichen Dokumentation“ sowie „Umgang mit Wertung“. Danach werden Kompetenzprofile über die verschiedenen Teilkompetenzen hinweg beschrieben und das Zusammenspiel der Teilkompetenzen für eine Beschreibung der Gesamtkompetenz in der Wahrnehmung von Unterrichtsaufzeichnungen diskutiert (Kap. 8.1.2). Abschließend werden die Analysen verschiedener Teilstichproben miteinander verglichen und berichtet, wie sich Personen entsprechend ihrer unterschiedlichen Erfahrungshintergründe in Bezug auf die Wahrnehmung von Unterricht in ihren Analysen von Unterrichtsaufzeichnungen unterscheiden (Kap. 8.2). Die Arbeit schließt mit einer Zusammenfassung und Diskussion der Ergebnisse (Kap. 9).

Die Arbeit besticht durch die fundierte Verbindung von Theorie und Empirie. Um die Kompetenz in der Unterrichtswahrnehmung erfassen und beschreiben zu können, entwickelt Schwindt in ihrer Arbeit ein sowohl empirie- als auch theoriegeleitetes „Gesamtmodell der kompetenten Unterrichtsanalyse“ (S. 74). Dabei stützt sie sich zum einen auf Ergebnisse aus der Beobachtungsforschung und leitet aus diesen Ergebnissen Kriterien ab, die ,einzelne Teilkompetenzen in der Wahrnehmung von Unterrichtsaufzeichnungen abbilden“ (S. 74). Zum anderen werden diese Teilkompetenzen durch Befunde aus der Expertiseforschung zu Wahrnehmungsprozessen von Lehrpersonen inhaltlich weiter ausdifferenziert. Dieses Gesamtmodell bildet die Basis dieser innovativen empirischen Untersuchung, deren Ziel es ist, aufzuzeigen, durch welche Ausprägungen des entwickelten Modells der kompetenten Unterrichtsanalyse sich die Kompetenzen von Lehrpersonen in der Wahrnehmung von Unterrichtsaufzeichnungen beschreiben lassen (S. 74) und welche Kompetenzprofile personenbezogen über die verschiedenen Teilkompetenzen hinweg identifiziert werden können (S. 75). Darüber hinaus untersucht Schwindt die Abhängigkeit dieser Kompetenzen von unterschiedlichem theoretischen Wissen sowie praktischen Erfahrungen und kann - bezogen auf die bereits genannten Teilkompetenzen - zeigen, inwiefern sich die jeweiligen Erfahrungshintergründe unterschiedlich auf die Analyse von Unterrichtsaufzeichnungen niederschlagen.

Aufgrund der soliden Anlage der Untersuchung und den teilweise sehr überraschenden und bedenkenswerten Ergebnissen gibt diese Arbeit nicht nur wertvolle Hinweise auf die Ausprägung und Entwicklung relevanter Komponenten der Professionalität von Lehrkräften. Sie bietet - so wie es die Autorin auch selbst anstrebt -fundierte Anschlussmöglichkeiten für die Überführung der Ergebnisse in ein Diagnoseinstrument zur Erfassung wahrnehmungsorientierter Kompetenzen von Lehrkräften. 


\section{Mammes, Denkmuster von Lehrkräften als Herausforderung für Unterrichtsent-}

wicklung. Auch der 2008 erschienenen Habilitationsschrift von Ingelore Mammes liegen wissensbezogene Konzepte der Professionalität von Lehrpersonen zugrunde. Der Schwerpunkt ihrer Untersuchung liegt auf unterrichtsbezogenen Lehrerkognitionen als Einflussgröße auf Unterrichtshandeln und Unterrichtsentwicklung. Entsprechend der Handlungs-/Entscheidungstheorie (vgl. Kraak u. Lindenlaub 1974), die die theoretische Basis der Arbeit darstellt, geht die Autorin davon aus, dass Lehrkräfte ihren Handlungsraum entsprechend zielgerichteter Entscheidungen aktiv-kognitiv strukturieren (S. 49).

In ihrer sowohl quantitativ als auch qualitativ angelegten empirischen Untersuchung geht die Autorin der Frage nach, inwiefern Lehrerkognitionen die Umsetzung von unterrichtsbezogenen Reformvorhaben beeinflussen können. Der inhaltliche Schwerpunkt liegt dabei auf dem naturwissenschaftlichen Unterricht und hier auf dem „Reformpostulat" (S. 8) einer naturwissenschaftlichen Grundbildung im Sinne der scientific literacy. Für Ingelore Mammes sind sowohl die Kenntnis bezüglich des normativ Gewünschten als auch eine positive Einstellung gegenüber diesem Gewünschten eine wichtige Voraussetzung dafür, dass unterrichtsbezogene Reformvorhaben umgesetzt werden. Vor diesem Hintergrund werden in der Arbeit „,beispielhaft Denkmuster von Lehrkräften der Naturwissenschaften zum Erwerb naturwissenschaftlicher Grundbildung“ (S. 8) untersucht. Auf diese Weise ,sollen Informationen gewonnen werden über das Passungsverhältnis gewünschter Unterrichtsrealisierung durch Reformverordnungen und die Handlungsbereitschaft der Lehrkräfte“ (S. 9). Die Arbeit knüpft damit an Studien zum Zusammenhang zwischen der Umsetzung von Reformbemühungen in Abhängigkeit von Kompetenzen, dem Engagement und den Überzeugungen von Lehrkräften an (vgl. Esslinger 2002 u.a.).

Die theoretische Einführung der Arbeit ist in sechs Kapitel untergliedert: Ausgehend von der den Schulleistungsvergleichsstudien TIMSS und PISA zugrunde liegenden gemeinsamen Konzeption naturwissenschaftlicher Grundbildung (Kap. 1) fasst die Autorin zunächst wesentliche aus diesen Studien resultierende Daten zur naturwissenschaftlichen Grundbildung zusammen. Die im internationalen Vergleich deutlich werdenden Defizite der naturwissenschaftlichen Grundbildung deutscher Schülerinnen und Schüler setzt sie in Beziehung mit Forschungsarbeiten, die für diese Defizite vor allem unterrichtsbezogene Erklärungsansätze anbieten (vgl. hier u.a. Stigler et al. 1996) (Kap.3). Die Erklärungsansätze werden anschließend durch die Darstellung verschiedener Lehr-/ Lernmodelle unterfüttert (Kap.4). Das Modell des pragmatisch-konstruktivistisch orientierten Lernens wird als normative Grundlage für die Reform des naturwissenschaftlichen Unterrichts vorgeschlagen. Im Anschluss daran nimmt Mammes die Neugestaltung des naturwissenschaftlichen Unterrichts aus der Perspektive verschiedener Unterrichtsentwicklungsansätze in den Naturwissenschaften in den Blick (Kap.5.1) und beschreibt in diesem Kontext die Implementierung des Faches „Natur und Technik“, das in den Jahren 2003 und 2004 an bayerischen Gymnasien flächendeckend in den Jahrgangsstufen 5 bis 7 eingeführt wurde (Kap. 5.2). Die Implementierung dieses Faches stellt den Untersuchungsrahmen der Arbeit dar. Abschließend widmet sich die Autorin dem Zusammenhang von schul-/unterrichtsbezogenen Reformvorhaben und der Lehrerpersönlichkeit (Kap.6). Aus den hier im Überblick dargestellten Forschungsergebnissen, in den auch bisher vorliegende Untersuchungen zu Vorstellungen oder Einstellungen von Lehrkräf- 
ten der Naturwissenschaften einbezogen sind, werden die Fragestellungen für das eigene Forschungsvorhaben abgeleitet (Kap. 7).

Nach der theoretischen Hinführung wird das methodische Vorgehen (in Kap. 8 und 9) beschrieben. Dabei werden zunächst in Anlehnung an Dann (2000) das Konstrukt „Lehrerkognitionen“ (Kap. 8.1) expliziert und der Fokus der Untersuchung (in Kap. 8.2 und 8.3) deutlich gemacht: Dieser liegt vor allem auf „Kognitionen [...], die originär-zielgerichtetes Handeln im Sinne der Unterrichtsausgestaltung umfassen“ (S. 55) und „die Ermittlung von Handlungsdispositionen zulassen“ (S. 52). Diese Handlungsdispositionen werden im Rahmen von schriftlichen Befragungen unter Zuhilfenahme von aus der Handlungs-/Entscheidungs-Theorie abgeleiteten Bedingungs- und Kontextfaktoren erhoben. Als Bedingungsfaktoren wählt die Autorin unterrichtsbezogene „Überzeugungen“ der Lehrkräfte, „persönliche Bilanzen“ sowie Methoden-,Kenntnis“ und „Methodenverständnis“, als Kontextfaktoren die Häufigkeit des „Einsatzes“ bestimmter Methoden sowie die ,berufliche Sozialisation“ der Lehrkräfte. In welcher Form diese Faktoren in Erhebungsitems für die schriftliche Befragung umgesetzt werden, stellt Ingelore Mammes im neunten Kapitel dar. Hier beschreibt die Autorin auch das an die quantitative Erhebung angeschlossene qualitative Vorgehen, mithilfe von Leitfadeninterviews die Ergebnisse der quantitativen Erhebung zu illustrieren, und skizziert die jeweiligen Stichproben.

Die Darstellung der Ergebnisse gliedert sich in zwei Kapitel (Kap. 10 und 11): Im zehnten Kapitel werden diejenigen Ergebnisse vorgestellt, die infolge der durch die schriftliche Befragung erfassten und mittels deskriptiver Statistik sowie multivariater Explorationstechniken ausgewerteten Daten gewonnen wurden. Dabei wird zunächst ein Überblick über die Strukturdaten der befragten Personen gegeben, anschließend werden entlang der formulierten Items die Ergebnisse zu den zuvor genannten Bedingungs- und Kontextfaktoren skizziert und es wird versucht, innerhalb dieser Faktoren „Musterläufigkeiten der Daten“ (S. 56) herauszuarbeiten, sodass Mammes für jeden Faktor verschiedene Gruppen von Lehrkräften generiert: so z. B. „reformorientierte“ und „traditionelle“ Lehrkräfte, „positiv“ bzw. „negativ Überzeugte“, „,demonstrationsorientierte Methodenkenner“ bzw. „gute Demonstrations- und eigentätige Methodenkenner“, „wenig demonstrations- und eigentätigkeitsbezogen Unterrichtende“ bzw. „demonstrations- und eigentätigkeitsbezogen Unterrichtende“ oder „früh“ und „spät Ausgebildete“ - um nur einige zu nennen. Schließlich wird überprüft, in welchen Zusammenhängen diese Gruppen miteinander stehen.

In Kapitel elf werden im Anschluss daran drei Fallbeispiele dargestellt, ,die die auf der Basis der statistischen Dateninterpretation gewonnenen Ergebnisse illustrieren“ (S. 56) und stellvertretend Gruppenzugehörigkeiten und Musterläufigkeiten erläutern sollen (vgl. S. 183 ff.).

In den abschließenden Kap. 12 und 13 werden die Ergebnisse der Untersuchung zusammengefasst, diskutiert und Schlussfolgerungen für Unterrichtsentwicklungsvorhaben gezogen.

Ingelore Mammes hat mit ihrer Studie den Versuch unternommen, aus den Denkmustern von Lehrkräften Impulse für unterrichtsbezogene Reformvorhaben zu gewinnen, und stellt sich damit einem für die Schul- und Unterrichtsentwicklungsforschung wichtigen Thema. Ihr sowohl quantitativ als auch qualitativ angelegtes Forschungsvorgehen verspricht dabei einen differenzierten Blick auf den Untersuchungsgegenstand. 
Dieses Potenzial wird in der Arbeit leider nicht vollständig ausgeschöpft. Der Schwerpunkt der Ergebnisdarstellung liegt auf einer zunächst rein deskriptiven Beschreibung der Ergebnisse durch Häufigkeiten entlang der Itemreihenfolge. Für die von der Autorin ausgewählten Bedingungs- und Kontextfaktoren wird ein durchweg ,differenziertes Datenbild“ (S. 136) resümiert, das allerdings kaum aussagekräftige Schlussfolgerungen zulässt. Um den deskriptiven Ergebnissen mehr Nachdruck zu verleihen, wäre es wünschenswert gewesen, die Datenreduzierung (Kap. 10.3) schon vorab vorzunehmen und sich so auf zentrale Punkte zu beschränken. Auch wenn die Rezensentinnen an manchen Stellen die aus den Ergebnissen gezogenen Ableitungen vor ihrem eigenen empirischen Hintergrund nicht teilen, ist es ebenso schade, dass die Erkenntnisse aus der Clusteranalyse (Kap. 10.4) und den Zusammenhangsanalysen (10.5) sowie deren theoriegeleitete Diskussion nicht mehr Raum einnehmen; denn hier verbirgt sich der eigentliche Gewinn der Arbeit, den Passungsgrad von Reformpotenzial und Reformpostulat zu beschreiben. So bleibt zu diskutieren, ob es sich bei den aufgezeigten Ergebnissen um repräsentative handlungsleitende Denkmuster handelt, die tatsächlich eine Herausforderung für die Unterrichtsentwicklung wären.

Hugener, Inszenierungsmuster im Unterricht und Lernqualität. Die gleichnamige schweizerisch-deutsche Videostudie unter der Leitung von E. Klieme, K. Reusser und C. Pauli bildet die Ausgangsbasis der ebenfalls 2008 erschienenen Dissertation von Isabelle Hugener. Die Verfasserin zeichnet in ihrer Arbeit ein Bild des Mathematikunterrichts in Deutschland und der Schweiz. Hierfür identifiziert sie in einem ersten Schritt zunächst Inszenierungsmuster von Unterricht, die anschließend in einem zweiten Untersuchungsschritt mit der subjektiv wahrgenommenen Lernqualität der Schülerinnen und Schüler in Verbindung gebracht werden. Drittens geht die Autorin der Frage nach, ob externe Beobachter das Unterrichtsmerkmal „,kognitive Aktivierung“ in den identifizierten Inszenierungsmustern unterschiedlich einschätzen. Schließlich vergleicht sie in einem vierten Analyseschritt ihre bisherigen Ergebnisse mit den Leistungsdaten der Schülerinnen und Schülern aus einem inhaltsspezifischen Nachtest.

Aus diesen zugrunde gelegten Untersuchungsschritten ergibt sich der Aufbau der Studie: Die theoretischen Überlegungen werden zunächst in drei eigenständige Kapitel untergliedert und ausgeführt. Nach der Einleitung wird im zweiten Kapitel ausgehend von einem aus der Lehr-/Lernpsychologie stammenden Lernbegriff der für die Studie relevante Theorierahmen des verstehensorientierten, aktiven Lernens und des Problemlösens abgesteckt. Hierbei beschränkt sich die Autorin nicht auf die kognitiven Aspekte (Kap. 2.1 bis 2.4) sondern widmet sich auch den motivationalen Bedingungen des Lernens und Problemlösens (Kap.2.5) genauso wie den Emotionen während des Lernens und Problemlösens (Kap. 2.6). Im dritten Kapitel überträgt Isabelle Hugener ihre Erkenntnisse auf den Mathematikunterricht. Hier betont die Autorin, dass der aus Sicht der Fachdidaktik zu bevorzugende problemlösende Unterricht durch erklärendes Lehrerhandeln im Unterrichtsgeschehen ergänzt werden muss. Fußend auf Aeblis Definition des problemlösenden Unterrichts beschreibt die Autorin (in Kap.31 und 3.2) zwei Weiterentwicklungen: zum einen die Differenzierung zwischen Lehrtätigkeit der Lehrperson und den Lernfunktionen der Schülerinnen und Schüler und zum anderen die von der Mathematikdidaktik präferierte individuelle, selbstregulierte Problemlöseaktivität. Aus 
dieser Zusammenschau und den zentralen Merkmalen eines problemlösenden Unterrichts (Kap.3.4) entwickelt die Autorin ihr Beobachtungsinstrument zur Analyse des mathematischen Inhalts und der Funktionen im Lernprozess sowie der darauf aufbauenden Inszenierungsmuster (Kap. 3.5).

Im vierten Kapitel steht die Frage nach der Wirksamkeit des problemlösenden Mathematikunterrichts im Mittelpunkt und die beschriebenen theoretischen Postulate werden für die empirische Überprüfung aufbereitet. Dabei geht die Autorin von den gesicherten Ergebnissen der Prozess-/Produkt-Forschung (Helmke u. Schrader 2001 u.a.) aus (Kap. 4.1) und ergänzt diese durch die fachdidaktisch orientierte Forschung zum Mathematikunterricht (Kap. 4.2) zunächst auf Basis von kleineren Interventionsstudien (Kap.4.2.1) und anschließend mit Studien zum Zusammenhang zwischen problemlösendem Mathematikunterricht und den Leistungen der Schülerinnen und Schüler (Kap.4.2.2).

Ein weiteres theoretisches Kapitel widmet die Autorin dem Inszenierungsmuster als Gestaltungsmerkmal im Unterrichtsverlauf (Kap. 5, S. 83): „Unter Inszenierungsmustern werden [...] die Sichtungsstrukturen [...] bzw. die beobachtbaren Oberflächenformen und -merkmale der Unterrichtsgestaltung [...] verstanden, dazu gehören einerseits die Sozialformen, andererseits die inhaltsbezogenen Lehrer- und Schüleraktivitäten in ihrer Anordnung und Kombination im zeitlichen Verlauf einer Unterrichtseinheit sowie ihrer Funktion im Lernprozess." Nach dieser begrifflichen Klärung und der Darlegung der didaktischen Basis (Kap. 5.1 und 5.2) bettet die Autorin ihre Studie in die videogestützte TIMSS-Unterrichtsforschung von 1995 und 1999 ein und erläutert länderspezifische Inszenierungsmuster (Kap.5.3). Dabei wird deutlich, dass sich die länderspezifischen Inszenierungen im Vergleich zwischen deutschen und schweizerischen Lehrkräften egalisieren und Unterschiede zwischen den Inszenierungsmustern durch pädagogische Theoriekulturen und nicht durch die Nationalität der Lehrkräfte erklärbar sind (Kap. 5.4). Nachdem die Methodik zur Identifikation von Identifizierungsmustern (Kap. 5.5) erläutert wurde, werden abschließend die Zusammenhänge zwischen der kognitiven Aktivierung, der Leistung und den Lernprozessmerkmalen mit den Inszenierungsmustern dargestellt (Kap.5.6 und 5.7). Der genaue Wortlaut der Fragestellungen zu den oben genannten vier Analyseschritten wird in Kapitel sechs dokumentiert.

Die Methodenkombination aus qualitativen und quantitativen Methoden in einem zyklischen Verfahren wird im siebten Kapitel beschrieben. Hierbei erläutert die Autorin die Besonderheiten der videogestützten Unterrichtsforschung im methodischen Kontext ihrer Studie, skizziert die Stichprobe, die Datenerhebung und diskutiert die Erfüllung der Gütekriterien für ihre Studie.

Die sich anschließende Ergebnisdarstellung orientiert sich an den im sechsten Kapitel formulierten Fragestellungen und beginnt mit der Analyse und Beschreibung der videografierten Unterrichtseinheiten. Auf dieser Basis werden Inszenierungsmuster identifiziert (Kap. 8.1 bis 8.5). Die gefundenen Inszenierungsmuster werden mit den subjektiv wahrgenommenen Lernprozessmerkmalen, der von Beobachtern eingeschätzten kognitiven Aktivierung und dem Leistungsstand der Schülerinnen und Schüler in Verbindung gebracht. Diese Zusammenhänge werden jeweils getrennt nach den analysierten Einführungsmustern (Kap. 8.6) und den Übungsmustern (Kap. 8.7) im Unterrichtsverlauf gesondert in den Blick genommen. Danach leitet Isabelle Hugener aus den Ergebnissen ihrer Studie Wirkanalysen (Kap. 8.8) ab. 
Im abschließenden Diskussionskapitel werden die zentralen Befunde zusammengefasst (Kap.9.1): Es werden die analysierten Inszenierungsmuster in ihrer Vielfalt dargestellt, Besonderheiten hinsichtlich des Mathematikunterrichts in den beiden Ländern Schweiz und Deutschland, der Schulform, der Replikation aus anderen Studien und der Lernqualität gezeigt. Im darauf folgenden Abschnitt (Kap.9.2) werden die verwendeten Analyseinstrumente auf der Basis der gewonnen Ergebnisse reflektiert, um aus diesen Überlegungen Schlussfolgerungen für weitere Ausführungen (Kap.9.3) ziehen zu können. Schlussendlich widmet sich das letzte Kapitel den Folgerungen für die pädagogische Praxis (Kap.9.4).

Detailliert werden in der vorliegenden Studie die pädagogisch-psychologischen Begrifflichkeiten wie Lernen und Problemlösen sowie die theoretischen Zusammenhänge zur videogestützten Unterrichtsforschung aufbereitet. Durch diese genaue Verortung im theoretischen Rahmen macht die Autorin deutlich, wie sie aus ihrem Datenmaterial Inszenierungsmuster extrahiert und diese dann mit den weiteren von ihr gewählten Variablen in Verbindung setzt. Dabei überzeugt die Darstellung der Wirkanalysen: Die im Datenmaterial gefundenen Einführungs- und Übungsmuster der Unterrichtssequenzen werden hier jeweils mit der Lernqualität, den wahrgenommenen Lernprozessmerkmalen, der eingeschätzten kognitiven Aktivierung und dem Leistungsstand methodisch präzise in Beziehung gesetzt. Umsichtig schätzt Isabelle Hugener die Belastbarkeit ihrer Stichprobe und damit die Tragfähigkeit der gefunden Zusammenhänge ein (S. 233): „Aufgrund der eher kleinen und unausgewogenen Stichprobe müssen einige Ergebnisse vorsichtig interpretiert werden. Da die Teilnahme an der videogestützen Unterrichtsstudie für Lehrpersonen mit erheblichem Aufwand verbunden war, erfolgte die Rekrutierung [...] auf freiwilliger Basis [...]. Darüber hinaus besteht eine Konfundierung zwischen Schulform und Land, weil die Schulformen in beiden Ländern nicht ausgewogen verteilt sind. [...] Mit 39 Klassen ist die Stichprobe für mehrebenenanalytische Stichproben relativ klein, was möglicherweise zu instabilen Ergebnissen führen kann.“" Es gelingt ihr durch ihre umsichtige Interpretation nicht nur, wesentliche Inszenierungsmuster von Mathematikunterricht zu identifizieren, sondern auch die Übertragbarkeit ihrer Ergebnisse auf anderen Fachunterricht anzuregen. Umso mehr enttäuscht, dass das Kapitel „Folgerungen für die pädagogische Praxis“" so knapp ausfällt und sich Anregungen für die pädagogische Praxis nur implizit finden. Zudem zieht die Autorin, obwohl in den Anfangskapiteln (Kap. 2 bis 5) ein weites Forschungsfeld aufbereitet wird, aus ihren Ergebnissen keine Schlussfolgerungen für die weitere Theoriebildung.

Isabelle Hugener hat ausgehend von gefilmten Daten im Mathematikunterricht eine Methode zur Identifikation von Inszenierungsmustern vorgelegt, die ebenso für anderen Fachunterricht fruchtbar gemacht werden kann. Die Kombination dieser analysierten Inszenierungsmuster mit lernrelevanten Faktoren klärt einige Zusammenhänge zwischen Unterricht und Lernerfolg auf, sodass sich dieses Vorgehen auch für andere Unterrichtssituationen bewähren könnte. Deshalb bietet die vorliegende Studie vielfache Anknüpfungspunkte für weitere Unterrichtsforschung. Weiterführende Schlussfolgerungen für die Unterrichtstheorie und die pädagogische Praxis dürften dann ebenfalls zu erwarten sein. 
Alle drei vorgestellten Studien möchten der Forschung im Bereich Schul- und Unterrichtsentwicklung neue Impulse geben. Obwohl die Autorinnen unterschiedliche Fragestellungen verfolgen, können die vorliegenden Untersuchungen in eine sich ergänzende Reihung gebracht werden: Ingelore Mammes zeichnet ein sehr facettenreiches Bild der Unterrichtswahrnehmung aus der Perspektive der Lehrkräfte. Darauf aufbauend kann die Arbeit von Katharina Schwindt gelesen werden, die nach Kriterien der kompetenten Unterrichtswahrnehmung fragt. Beide Autorinnen stellen die Innensicht des Unterrichts aus dem Blickwinkel von aktiv am Unterricht Teilnehmenden ins Zentrum ihrer Untersuchungen. Als Ergänzung hierzu kann die Arbeit von Isabelle Hugener gesehen werden, die durch die Analyse von videografiertem Unterricht die Außenperspektive liefert. Diese Innen- und Außensicht des Unterrichts zu kombinieren, scheint womöglich ein aussichtsreicher Weg, um Unterricht komplex beschreiben und Anregungen für Unterrichtsentwicklung gewinnen zu können.

Open Access Dieser Artikel unterliegt den Bedingungen der Creative Commons Attribution Noncommercial License. Dadurch sind die nichtkommerzielle Nutzung, Verteilung und Reproduktion erlaubt, sofern der/die Originalautor/en und die Quelle angegeben sind.

\section{Literatur}

Dann, H.-D. (2000). Lehrerkognition und Handlungsentscheidungen. In M. K. W. Schweer (Hrsg.), Lehrer-Schüler-Interaktionen. Pädagogisch-Psychologische Aspekte des Lehrens und Lernens in der Schule (S. 79-109). Opladen: Leske + Budrich.

Esslinger, I. (2002). Berufsverständnis und Schulentwicklung: ein Passungsverhältnis? Eine empirische Untersuchung zu schulentwicklungsrelevanten Berufsauffassungen von Lehrerinnen und Lehrern. Bad Heilbrunn: Klinkhardt.

Helmke, A., \& Schrader, F.-W. (2001). Determinanten der Schulleistung. In D. H. Rost (Hrsg.), Handwörterbuch Pädagogische Psychologie (2. überarbeitete und erweitere Auflage, S. 81-91). Weinheim: Beltz PVU.

Kraak, B., \& Lindenlaub, S. (1974). Entwurf einer Handlungs-Entscheidungstheorie. Mitteilungen und Nachrichten des Deutschen Instituts für Internationale Pädagogische Forschung, (75/76), 93-105.

Stigler, J. W., Gonzales, P. A., Kawanka, T., Knoll, S., \& Serrano, A. (1996). The TIMSS videotape classroom study. Methods and findings from an exploratory research project on eights-grade mathematics instruction in Germany, Japan and the United States. Washington, DC: Government Printing Office. 